\title{
An Alternative Fuzzy EOQ Model with Backlogging for Selling Price and Promotional Effort Sensitive Demand
}

\author{
Sujit Kumar De · Shib Sankar Sana
}

Published online: 26 October 2014

(C) Springer India Pvt. Ltd. 2014

\begin{abstract}
This paper deals with a classical Economic Order Quantity model with backlogging and the demand of end customers is dependent on promotional effort and selling price simultaneously. The order quantity and shortage quantities are functionally related to promotional effort (PE), unit selling price and time alone. At first, a crisp solution is made. Then, a general fuzzy optimal and intuitionistic fuzzy optimal policy have been discussed, considering selling price and PE as fuzzy number and fuzzy variable respectively. Finally, a graphical illustration, numerical examples with sensitivity analysis and conclusion are made for justification of the model.
\end{abstract}

Keywords Backlogging - Inventory · Fuzzy selling price · Fuzzy promotional effort · Index $\cdot \alpha$-cut $\cdot$ Intuitionistic fuzzy technique

\section{Introduction}

The Economic Order Quantity (EOQ) model is an elegant formula which is used in the supply chain (Cárdenas-Barrón [1], Cárdenas-Barrón et al. [2,3], Cárdenas-Barrón and TreviñoGarza [4], Cárdenas-Barrón and Porter [5]; Sarkar [6-8], Sarkar et al. [9],Sett et al. [10]). For any kind of inventory, we generally know that the demand rate is basically depends upon the intensity of publicity/promotional effort and the selling price of unit items. A considerable selling price per unit item may be the cause of high demand and it may act as motivational factor over unit selling price. The demand is also affected by PE such as advertising, sales teams initiatives, better services, free gifts, delay in payment, etc. Among others, researchers like Sana et al. [11], Sana [12-15], Cárdenas-Barrón and Sana [16],

S. Kumar De

Department of Mathematics, Midnapore College, Medinipur (W), W. B., India

e-mail: skdemamo2008.com@gmail.com

S. S. Sana $(\varangle)$

Department of Mathematics, Bhangar Mahavidyalaya, South 24 Parganas, Bhangar 743502, India

e-mail: sana_sankar@yahoo.co.in 
Sana [17] and Sarkar et al. [18,19] did research works in this direction. Sarkar and Sarkar [20] extended an inventory model for deteriorating items with stock-dependent demand, considering time varying backlogging rate as well as time varying deterioration rate. Pal et al. [21] investigated two echelon production inventory model where selling price and ordering size are decision variables. De et al. [22] suggested an EOQ model for Phase Inventory system with induced demand and periodic cycle length. Sana [23] analyzed an EOQ model for uncertain demand for limited capacity of own warehouse, and the rented warehouse is considered, if needed. Sana and Goyal [24] proposed a (Q, r, L) model for stochastic demand with lead-time dependent partial backlogging.

In this competitive world no other variable is finite rather they are non-randomly uncertain in nature. For this reason, we consider PE as fuzzy interval. On the other hand, the selling price may lie in a fuzzy set. Zadeh [25] first developed the concept of fuzzy set theory and later on, Bellman and Zadeh [26] made an application of fuzzy set theory in several decision making problems of operations research. Since then, numerous research findings have been established along this direction. Kaufmann and Gupta [27] developed a fuzzy mathematical model in engineering and management science. Vojosevic et al. [28] fuzzified the order cost into trapezoidal fuzzy number in the backorder model. Using these propositions, Wu and Yao [29] studied a fuzzy inventory with backorder for fuzzy order quantity and fuzzy shortage quantity. With the help of fuzzy extension principle, an EOQ in fuzzy sense for inventory without backorder model had been developed by Lee and Yao [30]. Yao et al. [31] analyzed a fuzzy model without backorder for fuzzy order quantity and fuzzy demand quantity. Kao and Hsu [32] developed a lot size reorder point inventory model with fuzzy demands by considering the $\alpha$-cut of the fuzzy numbers and they used ranking index to solve the model. De et al. [33] developed an EPQ (Economic Production Quantity) model for fuzzy demand rate and fuzzy deterioration rate using the $\alpha$-cut of the membership function of the fuzzy parameters. De et al [34] studied an economic ordering policy of deteriorated items with shortage and fuzzy cost coefficients for vendor and buyer. Recently, Kumar et al. [35] developed a fuzzy model with ramp type demand rate and partial backlogging.

In crisp sense, several optimization techniques have been used in the modern developed inventory model. Some of these are, Golden Region Search method in Simulation technique that was developed by Kabirian and Olafsson [36] and another one is an analytic approach via Eigen values of the system Jacobian Matrix expressing from characteristic polynomial which was analyzed by Saleh et al. [37]. But, in fuzzy environment, the early stage of Intuitionistic Fuzzy Set (IFS) was developed by Atanassov [38]. The concept of IFS can be viewed as an alternative approach to define a fuzzy set while the information available in the system is not sufficient for the definition of an imprecise concept or ambiguity (vagueness) concerning the description of the semantic meaning of declaration of statements relating to an economic world in the sets itself. Therefore, it is to be expected that IFS can be used to simulate human decision making process and activities that requires human expertise and knowledge which is valid and reliable. Here, the degree of rejection and satisfaction are considered so that the sum of both values is always less than unity. Angelov [39] implemented the IFS for optimizing the real world problem in intuitionistic fuzzy environment. An interval valued IFS was developed by Atanassov and Gargov [40] and, a solution of a probabilistic fixed order interval system was analyzed by Banerjee and Roy [41]. De and Sana [42] proposed the Classical EOQ model for promotional effort sensitive demand which was considered as intuitionistic fuzy variable. De and Sana [43] developed the EOQ model with backordering for promotional effort and selling price sensitive demand, introducing intuitionistic fuzzy approach. Recently, De et al. [44] established an interpolating by pass to Pareto optimality for the EOQ model with time sensitive backlogging, applying intuitionistic fuzzy technique. In intuitionistic fuzzy 
environment, De and Sana [45] presented a global optimal solution for the multi-periods production-inventory model with capacity constraints for multi-manufacturers. Das et al. [46] investigated a backorder EOQ model for natural leisure/closing time system while the demand rate depends upon the total shortage period under the seasonal effect.

In this paper, we have used PE and unit selling price as IFS. However, the demand rate is considered such a way that (i) one part depends upon promotional effort and (ii) the other part goes to infinity when the unit selling price reaches to a certain minimum bound and reaches zero when the unit selling price attains its upper bound. Considering fuzzy as well as intuitionistic fuzzy environment, we have developed the model under proposed considerations. First, we have optimized the profit function under crisp environment. Thereafter, we have constructed a General Fuzzy Optimization (GFO) problem and Intuitionistic Fuzzy Optimization (IFO) problem. Using the $\alpha$-cuts of the membership functions and $\beta$-cuts of the non-membership functions for the fuzzy sets, we have obtained some numerical results via some algorithms. A sensitivity analysis for Crisp, GFO and IFO models, graphical illustrations and a concluding remark are made to generalize the model.

\section{Assumptions and Notations}

The following notations and assumptions are used to develop the model.

\section{Notation}

$\begin{array}{ll}q: & \text { The order quantity per cycle. } \\ D: & \text { Demand rate per year. } \\ \mathrm{s}: & \text { Shortage quantity per cycle. } \\ c_{1}: & \text { Setup cost }(\$) \text { per cycle. } \\ c_{2}: & \text { Inventory holding cost }(\$) \text { per unit quantity per cycle. } \\ c_{3}: & \text { Shortage cost }(\$) \text { per unit quantity per unit time. } \\ s_{1}: & \text { Selling price }(\$) \text { per unit item. } \\ s_{1 \text { max }}: & \text { Upper bound of selling price }(\$) \text { per unit item. } \\ s_{1 \text { min }}: & \text { Lower bound of selling price }(\$) \text { per unit item. } \\ \rho: & \text { Promotional effort per unit time. } \\ \rho_{\max }: & \text { Upper bound of promotional effort } \rho . \\ \rho_{\min }: & \text { Lower bound of promotional effort } \rho . \\ p_{1}: & \text { Purchasing price }(\$) \text { of unit item. } \\ k: & \text { Promotional cost }(\$) \text { per factor over promotional effort. } \\ m: & \text { Elasticity constant, a positive integer. } \\ t_{1}: & \text { Shortage period (months). } \\ t_{2}: & \text { Inventory run time (months). } \\ T: & \text { Cycle time in months. } \\ \mathrm{Z}: & \text { Average profit (\$) of the inventory. }\end{array}$

\section{Assumptions}

1. Replenishment rate is instantaneous but its size is finite.

2. The time horizon is finite (months)

3. Shortages are allowed

4. Demand rate is unit selling price $\left(s_{1}\right)$ and promotional effort $(\rho)$ dependent where $D\left(s_{1}, \rho\right)=\eta\left(\frac{s_{\max }-s_{1}}{s_{1}-s_{\min }}\right)+\tau\left(\frac{\rho}{1+\rho}\right), \quad \eta$ and $\tau$ are constants. Quite often, the demand rate increases with promotional effort $(\rho)$ and it decreases with selling price $\left(s_{1}\right)$. 


\subsection{Crisp Mathematical Model}

In crisp model, inventory starts with shortages due to lead time or any other factors and it continues up to time $t_{1}$. When the ordering quantity (q) reaches at time $t_{1}$, the backlogged amount (s) is adjusted. Then, the rest amount (q-s) satisfies the demand for the period $\left(0, t_{2}\right)$ and it reaches to zero level at the end of time $t_{2}$. Therefore, $s=D t_{1},(q-s)=D t_{2}$ and the total cycle length is $T=t_{1}+t_{2}=q / D$. The average profit per cycle, by trading of purchasing cost, set up cost, inventory cost, shortage cost, promotional cost and sales price, is

$$
Z=\left(s_{1}-p_{1}\right) D-\frac{c_{1}}{T}-\frac{c_{2} D t_{2}^{2}}{2 T}-\frac{c_{3} D t_{1}^{2}}{2 T}-k \rho^{m}
$$

where

$$
\frac{s}{t_{1}}=\frac{q-s}{t_{2}}=\frac{q}{T}=D, T=t_{1}+t_{2}
$$

Now, substituting the formulas of Eq. (2) in Eq. (1), we have

$$
\text { Maximize } Z=\left(s_{1}-p_{1}\right) D-\frac{c_{1} D}{q}-\frac{c_{2}(q-s)^{2}}{2 q}-\frac{c_{3} s^{2}}{2 q}-k \rho^{m}
$$

subject to the conditions

$$
s=D t_{1}, q=D T, T=t_{1}+t_{2}
$$

where

$$
D\left(s_{1}, \rho\right)=\eta\left(\frac{s_{1 \max }-s_{1}}{s_{1}-s_{1 \text { min }}}\right)+\tau\left(\frac{\rho}{1+\rho}\right)
$$

Now, our objective is to maximize $\mathrm{Z}$ where $\rho, q$ and $s$ are the decision variables.

\subsection{Fuzzy Mathematical Model}

In the traditional EOQ model, we have seen that, the demand rate is constant. In practice, in many cases, it depends upon PE and unit selling price which are flexible in nature. For this reason, we shall fuzzify those.

Now, using Eqs. (3), (4) and (5), the fuzzy problem for Eq. (3) is

$$
\text { Maximize } \tilde{Z}=\left(\tilde{s}_{1}-p_{1}\right) \tilde{D}-\frac{c_{1}}{T}-\frac{c_{2} \tilde{D} t_{2}^{2}}{2 T}-\frac{c_{3} \tilde{D} t_{1}^{2}}{2 T}-k \tilde{\rho}^{m}
$$

Subject to the conditions

$$
\tilde{s}=\tilde{D} t_{1}, \tilde{q}=\tilde{D} T, T=t_{1}+t_{2}
$$

Where

$$
\tilde{D}\left(s_{1}, \rho\right)=\eta\left(\frac{s_{1 \max }-\tilde{s}_{1}}{\tilde{s}_{1}-s_{1 \min }}\right)+\tau\left(\frac{\tilde{\rho}}{1+\tilde{\rho}}\right) .
$$

\subsubsection{Cases of Optimality}

Now, we have to solve (6) for the following cases:

We may assume $s_{1} \in\left[s_{1 \min }, s_{1 \max }\right]$ and $\rho \in\left[\rho_{\min }, \rho_{\max }\right]$.In our proposed model, we observe that the demand rate tends to infinite when $s_{1} \rightarrow s_{1 \mathrm{~min}}$, and the first part of the demand rate tends to zero when $s_{1} \rightarrow s_{1 \max }$. Also, it is observed that the crisp optimality 
attains at $\rho=\rho^{*}$ (say), and it attains its maximum value (when average profit reaches near zero) at $\rho=\rho_{\max }$. Thus, we take the domain space for $s_{1}$ and $\rho$ as follows:

1. $s_{1} \in\left[s_{1 \min }, s_{1 \max }\right]$ and $\rho \in\left[\rho_{\min }, \rho^{*}\right]$

2. $s_{1} \in\left[s_{1 \min }, s_{1 \max }\right]$ and $\rho \in\left[\rho^{*}, \rho_{\max }\right]$

If we seek to split more sub partitions of $\left[\rho^{*}, \rho_{\max }\right]$, taking any intermediate value at $\rho=\rho^{\prime}$, then

3. $s_{1} \in\left[s_{1 \text { min }}, s_{1 \max }\right]$ and $\rho \in\left[\rho^{*}, \rho^{\prime}\right]$

4. $s_{1} \in\left[s_{1 \text { min }}, s_{1 \text { max }}\right]$ and $\rho \in\left[\rho^{\prime}, \rho_{\max }\right]$.

Now, to obtain a general fuzzy optimal solution (GFO) of Eq. (6), we assume PE $(\rho)$ and unit selling price $\left(s_{1}\right)$ as L-fuzzy and the objective function as R-fuzzy numbers. Then the membership functions for $\rho, s_{1}$ and $\mathrm{z}$ are obtained as follows:

$$
\begin{aligned}
& \mu_{\tilde{\rho}}(\tilde{\rho})=\left\{\begin{array}{lll}
0 & \text { for } & \rho>\rho_{0} \\
\frac{\rho_{0}-\rho}{p_{01}} & \text { for } & \rho_{0}-p_{01} \leq \rho \leq \rho_{0} \\
1 & \text { for } \rho<\rho_{0}-p_{01}
\end{array}\right. \\
& \mu_{\tilde{s}_{1}}\left(\tilde{s}_{1}\right)=\left\{\begin{array}{lll}
0 & \text { for } s_{1}>s_{10} \\
\frac{s_{10}-s_{1}}{p_{02}} & \text { for } & s_{10}-p_{02} \leq \rho \leq s_{10} \\
1 & \text { for } & s_{1}<s_{10}-p_{02}
\end{array}\right.
\end{aligned}
$$

and

$$
\mu_{\tilde{z}}(\tilde{z})= \begin{cases}0 & \text { for } z<z_{0} \\ \frac{z-z_{0}}{p_{03}} & \text { for } \quad z_{0} \leq z \leq z_{0}+p_{03} \\ 1 & \text { for } z>z_{0}+p_{03}\end{cases}
$$

Using aspiration level $\alpha$ to each membership function, the equivalent crisp optimization problem can be obtained with the help of Bellman and Zadeh [24] and Zimmermann [47]. Therefore, applying the membership functions in Eqs. (9)-(11), the fuzzy non-linear function Eq. (6) may be transformed into crisp equivalent and is given by

$$
\begin{aligned}
& \text { Max } \alpha \\
& \text { subject to } z>z_{0}+\alpha p_{03}
\end{aligned}
$$

where

$$
\begin{aligned}
z= & {\left[\left(s_{10}-\alpha p_{02}-p_{1}\right)-\frac{c_{2} t_{2}^{2}}{2 T}-\frac{c_{3}\left(T-t_{2}\right)^{2}}{2 T}\right] } \\
& {\left[\eta \frac{s_{1 \text { max }}-s_{10}+\alpha p_{02}}{s_{10}-\alpha p_{02}-s_{1 \text { min }}}+\tau \frac{\rho_{0}-\alpha p_{01}}{1+\rho_{0}-\alpha p_{01}}\right]-\frac{c_{1}}{T}-k\left(\rho_{0}-\alpha p_{01}\right)^{m} }
\end{aligned}
$$

\subsubsection{Formulation of Intuitionistic Fuzzy Optimization (IFO) Technique}

Intuitionistic Fuzzy Set is generally applied when the sufficient information in the fuzzy set is not available or a lack of knowledge in fuzzy decision making process exists. In such cases, the IFS is a proper subset of the fuzzy set in which the degree of rejection (non-membership) and the degree of acceptance (membership) are defined simultaneously and they are not complementary to each other. In fuzzy set, our aim is to maximize the support (degree of acceptance) of the membership function that results in minimizing the height of negation or 
non-membership (degree of rejection). Thus, we may transform the optimization problem into the following way:

$$
\begin{array}{ll}
\text { Max } & \mu(\bar{X}) \quad \bar{X} \in R^{+} \\
\text {Min } & v(\bar{X}) \quad \bar{X} \in R^{+} \\
& v(\bar{X}) \geq 0 \\
\text { Subject to } & \mu(\bar{X}) \geq v(\bar{X}) \\
& \mu(\bar{X})+v(\bar{X})<1, \bar{X} \in R^{+}
\end{array}
$$

where $\mu(\bar{X})$ denotes the degree of membership (acceptance) function of $\bar{X}$ to the IFS and $v(\bar{X})$ denotes the degree of non-membership (rejection) function of $\bar{X}$ for the IFS.

\subsubsection{IFO Technique for Solving the Objective Functions with Linear Membership and Non-membership Functions}

In this section, we define the membership functions of the objective functions with their lower and upper bounds. Let $L^{a c c}$ as lower and $U^{a c c}$ as upper bound of the objective function. These values are determined as follows: The lower and upper bounds of $\rho$ and $s_{1}$ are taken first. These predictions depend upon the decision maker's choice and expertise that is concerned with the insight of the model. These values are then arranged as a pair like $\left(\rho, s_{1}\right)=\{(\max , \max ),(\max$. $\min )$, (min. $\max ),(\min , \min )\}$. The optimum value of each objective function for each pair $(\rho)$, is to be found out first. Let $\bar{X}_{1}^{*}, \bar{X}_{2}^{*}, \bar{X}_{3}^{*} \ldots \ldots . \bar{X}_{j}^{*}$ be the respective optimal solutions to the objective function $Z\left(\bar{X}_{j}\right)$ for $j=1,2,3$ and 4 . For each objective, the lower bound $=\operatorname{Min}\left\{\mathrm{Z}\left(\bar{X}_{\mathrm{j}}\right)\right\}$ and the upper bound $U^{a c c}=\operatorname{Max}\left\{\mathrm{Z}\left(\bar{X}_{\mathrm{j}}\right)\right\}$. But, in IFO, the degree of rejection (non-membership) and degree of acceptance (membership) are considered so that the sum of both values is less than one. To obtain the membership functions under IFO environment let $L^{r e j}$ be the lower and $U^{r e j}$ be the upper bound of the objective functions $Z\left(\bar{X}_{j}\right)$ where $L^{a c c} \leq L^{r e j} \leq U^{r e j} \leq U^{a c c}$. These values are obtained from the following definition.

Definition 1 For maximization problem, the upper bound for non-membership function (rejection) is always less than that of the membership function (acceptance). We take lower bound and upper bound for non-membership function as follows:

$$
\begin{aligned}
& L^{r e j}=L^{a c c}+\lambda\left(U^{a c c}-L^{a c c}\right) \text { for } \lambda=0 \\
& U^{a c c}=U^{r e j}+\lambda\left(U^{a c c}-L^{a c c}\right) \text { for } 0<\lambda<1
\end{aligned}
$$

Definition 2 If we have to take the value of the parameter/variable as minimum, then the lower bound for non-membership function (rejection) is always greater than the membership function (acceptance). We take lower bound and upper bound for non-membership function as follows:

$$
\begin{aligned}
& L^{r e j}=L^{a c c}+\lambda\left(U^{a c c}-L^{a c c}\right) \text { for } 0<\lambda<1 \\
& U^{r e j}=U^{a c c}+\lambda\left(U^{a c c}-L^{a c c}\right) \text { for } \lambda=0
\end{aligned}
$$

Note that, for both the cases of definition-1 and definition-2, we always choose $\lambda$ in such a way that the differences between two consecutive parameters are minimum with realistic value. 
The linear membership function and linear non-membership function for the objective function $Z(\bar{X})$ are given by

$$
\begin{aligned}
& \mu(Z(\bar{X}))= \begin{cases}0 & \text { for } Z(\bar{X})<L^{a c c} \\
\frac{Z(\bar{X})-L^{a c c}}{U^{a c c}-L^{a c c}} & \text { for } L^{a c c} \leq Z(\bar{X}) \leq U^{a c c} \\
1 & \text { for } Z(\bar{X})>U^{a c c}\end{cases} \\
& v(Z(\bar{X}))= \begin{cases}1 & \text { for } Z(\bar{X})<L^{r e j} \\
\frac{U^{r e j}-Z(\bar{X})}{U^{r e j}-L^{r e j}} & \text { for } L^{r e j} \leq Z(\bar{X}) \leq U^{r e j} \\
0 & \text { for } Z(\bar{X})>U^{r e j}\end{cases}
\end{aligned}
$$

The linear membership and non-membership function of $\rho$ is given by

$$
\begin{aligned}
& \mu_{\rho}(\rho)= \begin{cases}0 & \text { for } \rho>\rho_{U}^{a c c} \\
\frac{\rho_{U}^{a c c}-\rho}{\rho_{U}^{a c c}-\rho_{L}^{a c c}} & \text { for } \rho_{L}^{a c c} \leq \rho \leq \rho_{U}^{a c c} \\
1 & \text { for } \rho<\rho_{L}^{a c c}\end{cases} \\
& v_{\rho}(\rho)= \begin{cases}1 & \text { for } \rho>\rho_{U}^{r e j} \\
\frac{\rho-\rho_{L}^{r e j}}{\rho_{U}^{r e j}-\rho_{L}^{r e j}} & \text { for } \rho_{L}^{r e j} \leq \rho \leq \rho_{U}^{r e j} \\
0 & \text { for } \rho<\rho_{L}^{r e j}\end{cases}
\end{aligned}
$$

The linear membership and non-membership function of $s_{1}$ is given by

$$
\begin{aligned}
& \mu_{s_{1}}\left(s_{1}\right)= \begin{cases}0 & \text { for } s_{1}>s_{1 U}^{a c c} \\
\frac{s_{1 U}^{a c c}-s_{1}}{s_{1 U}^{a c c}-s_{1 L}^{a c c}} & \text { for } s_{1 L}^{a c c} \leq s_{1} \leq s_{1 U}^{a c c} \\
1 & \text { for } s_{1}<s_{1 L}^{a c c}\end{cases} \\
& v_{s_{1}}\left(s_{1}\right)= \begin{cases}1 & \text { for } s_{1}>s_{1 U}^{r e j} \\
\frac{s_{1}-s_{1 L}^{r e j}}{s_{1 U}^{r e j}-s_{1 L}^{r e j}} & \text { for } s_{1 L}^{r e j} \leq s_{1} \leq s_{1 U}^{r e j} \\
0 & \text { for } s_{1}<s_{1 L}^{r e j}\end{cases}
\end{aligned}
$$

The notations used here keep their usual meanings. The diagrams of membership and nonmembership functions of the concerned variables are shown in Figs. 1, 2, 3.

Based on fuzzy decision of Bellman and Zadeh [19] together with membership and nonmembership functions of Eqs. (14)-(19) and Angelov's [32] approach of the IFO problem,

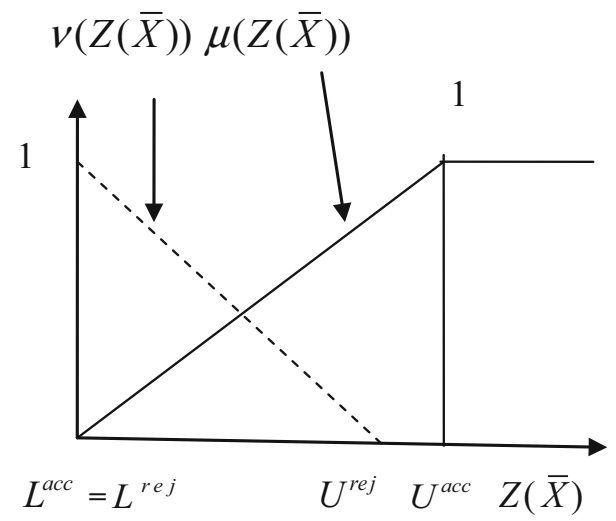

Fig. 1 (Non) Membership function for Z (R-fuzzy) 


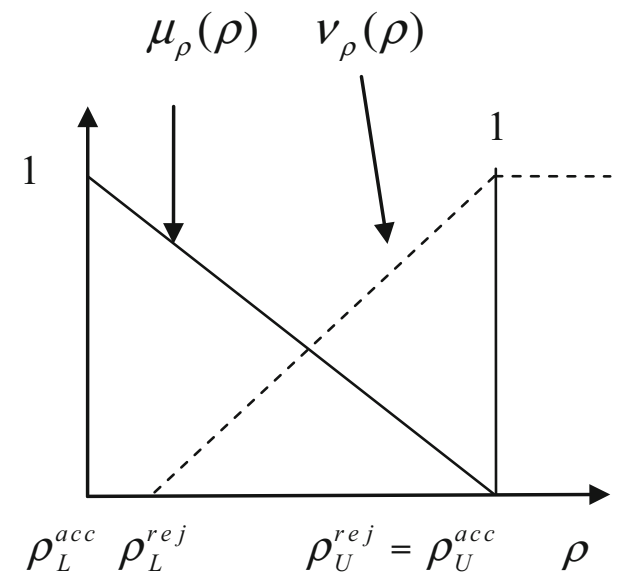

Fig. 2 (Non) Membership function for $\rho$ (L-fuzzy)

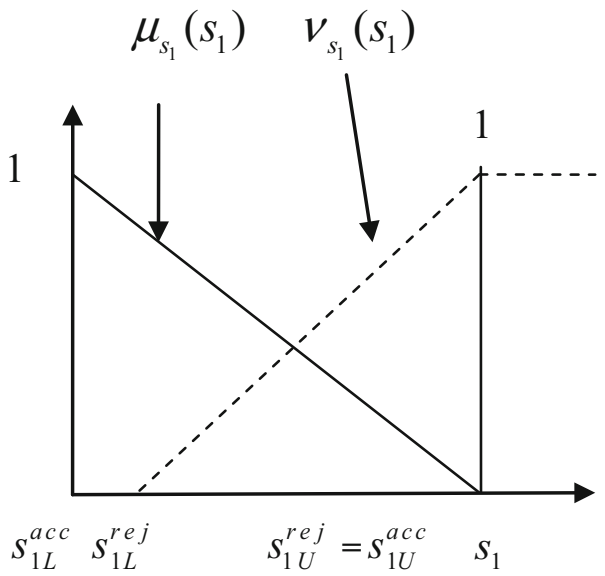

Fig. 3 (Non) Membership function for $s_{1}$ (L-fuzzy)

Eq. (13) can be written as follows

$$
\begin{array}{ll}
\text { Max } & \alpha-\beta \\
\text { Subject to } & Z(\bar{X}) \geq L^{a c c}+\alpha\left(U^{a c c}-L^{a c c}\right) \\
& Z(\bar{X}) \geq U^{r e j}-\beta\left(U^{r e j}-L^{r e j}\right) \\
& \beta \geq 0, \alpha \geq \beta, \alpha+\beta<1, \bar{X} \in R^{+} \\
& \quad \text { where } \bar{X}=\left(\rho, s_{1}, s, q, \alpha, \beta\right)
\end{array}
$$

\subsubsection{Computational Algorithm}

Step-1. Optimize the objective function for different fixed pair of values of $\left(\rho, s_{1}\right)$. Find the lower bound and upper bound of the objective function $L^{a c c}=\operatorname{Min}\left\{Z\left(\bar{X}_{j}\right)\right\}$ and the upper bound $U^{a c c}=\operatorname{Max}\left\{Z\left(\bar{X}_{j}\right)\right\}$ for $j=1,2,3$ and 4 .

Step-2. At first construct the membership functions of objective goals, promotional index $\rho$ and unit selling price $s_{1}$ with appropriate tolerances (decision maker's choice). 
Then, from Definition-1 and Definition-2, for suitable choice of $\lambda$ (requires decision maker's expertise and knowledge) find the non-membership functions of the objective function Z, promotional index $\rho$ and unit selling price $s_{1}$.

Step-3. Construct the fuzzy programming problem and its equivalent crisp optimization problem stated in Eq. (20).

Step-4. Use a compromised/ ideal solution of $\rho$ and $s_{1}$ for the problem stated in Eq. (20). Solve this non-linear programming problem with appropriate programming for the decision variable $\alpha$ and $\beta$ and then obtain $t_{1}^{*}, t_{2}^{*}, \rho^{*}, s_{1}^{*}, q^{*}, s^{*}, \alpha^{*}, \beta^{*}$ and $Z^{*}$.

Note: The compromise solution of $\rho$ and $s_{1}$ are given by

$$
\left\langle\begin{array}{l}
s_{1}^{*}=0.5\left(s_{1 L}^{r e j}+s_{1 U}^{a c c}\right)+0.5 \beta^{*}\left(s_{1 U}^{r e j}-s_{1 L}^{r e j}\right)-0.5 \alpha^{*}\left(s_{1 U}^{a c c}-s_{1 L}^{a c c}\right) \\
\rho^{*}=0.5\left(\rho_{L}^{r e j}+\rho_{U}^{a c c}\right)+0.5 \beta^{*}\left(\rho_{U}^{r e j}-\rho_{L}^{r e j}\right)-0.5 \alpha^{*}\left(\rho_{U}^{a c c}-\rho_{L}^{a c c}\right)
\end{array}\right\rangle
$$

\section{Numerical Example}

\subsection{Numerical Example-1}

For Crisp model, let a seller starts his/ her business with initial demand rate $\eta=50$ units, $\tau=70$ units, setup cost $c_{1}=\$ 28$, holding cost per unit item $c_{2}=\$ 20$, shortage cost $c_{3}=\$ 100, m=3$, selling price per unit item $s_{1}=\$ 20$, purchasing price $p_{1}=\$ 12$, $k=\$ 15$, the cycle time $T=0.4$ month. Then, we get the following results in Tables 1,2 , 3,4 .

From the above table, we see that the maximum profit attains at $\rho^{*}=3.667$ and $m^{*}=1$; the inventory run time is $\mathbf{1 2}$ days and the shortage period is 2 days only. Also, we further observe that the profit will be nominal for $m=3$ and $\rho_{\max }=3.6$.

Table 1 Crisp optimal solution for fixed $\rho$ and $\mathrm{m}$

\begin{tabular}{llllll}
\hline$q^{*}$ & $s^{*}$ & $\rho^{*}$ & $t_{2}^{*}$ days & $t_{1}^{*}$ days & $Z^{*}(\$)$ \\
\hline 65.364 & 10.894 & 1.216 & 10 & 2 & 665.617
\end{tabular}

Table 2 Crisp optimal solution for different $\rho$ and $\mathrm{m}$

\begin{tabular}{lllllll}
\hline$m$ & $\rho^{*}$ & $q^{*}$ & $s^{*}$ & $t_{2}^{*}$ days & $t_{1}^{*}$ days & $Z^{*}(\$)$ \\
\hline 1 & $\mathbf{3 . 6 6 7}$ & $\mathbf{7 2 . 0 0 0}$ & $\mathbf{1 2 . 0 0 0}$ & 10 & 2 & $\mathbf{7 1 5 . 0 0}$ \\
2 & 1.605 & 67.251 & 11.208 & & & 675.96 \\
3 & 1.216 & 65.364 & 10.894 & & & 665.62 \\
4 & 1.080 & 64.537 & 10.756 & & & 662.54 \\
5 & 1.017 & 64.119 & 10.687 & & & 661.72 \\
6 & 0.984 & 63.887 & 10.648 & & & 661.73 \\
7 & 0.965 & 63.748 & 10.625 & & & 662.07 \\
8 & 0.953 & 63.663 & 10.610 & & & 662.53 \\
9 & 0.946 & 63.609 & 10.601 & & & 663.03 \\
10 & 0.941 & 63.574 & 10.596 & & & 6 \\
\hline
\end{tabular}

Bold face indicates optimal solution 
Table 3 Crisp optimal solution for different shortage period

\begin{tabular}{llllll}
\hline $\mathrm{t}_{2}$ months & $\mathrm{t}_{1}$ months & $\rho^{*}$ & $q^{*}$ & $s^{*}$ & $Z^{*}(\$)$ \\
\hline 0.4 & 0.0 & 2.021 & 18.734 & 0.000 & 930.07 \\
.36 & .04 & 2.036 & 18.777 & 1.878 & 956.33 \\
$\mathbf{3 2}$ & $\mathbf{. 0 8}$ & $\mathbf{2 . 0 3 8}$ & $\mathbf{1 8 . 7 8 3}$ & $\mathbf{3 . 7 5 7}$ & $\mathbf{9 6 0 . 0 9}$ \\
.28 & .12 & 2.028 & 18.753 & 5.262 & 941.32 \\
.24 & .16 & 2.006 & 18.684 & 7.474 & 900.14 \\
.20 & .20 & 1.970 & 18.572 & 9.285 & 836.80 \\
.16 & .24 & 1.919 & 18.407 & 11.044 & 751.74 \\
.12 & .28 & 1.849 & 18.172 & 12.720 & 645.65 \\
.08 & .32 & 1.755 & 17.838 & 14.271 & 519.58 \\
.04 & .36 & 1.628 & 17.344 & 15.610 & 375.24 \\
.00 & .40 & 1.443 & 16.541 & 16.541 & 215.70
\end{tabular}

Bold face indicates optimal solution

Table 4 Crisp optimal solution for different $\rho$ and $s_{1}$

\begin{tabular}{|c|c|c|c|c|c|c|}
\hline $\begin{array}{l}s_{1} \\
12-15\end{array}$ & $\begin{array}{l}\rho^{*} \\
\ldots \ldots\end{array}$ & $\begin{array}{l}q^{*} \\
\ldots \ldots \ldots\end{array}$ & $\begin{array}{l}s^{*} \\
\ldots \ldots \ldots\end{array}$ & $\begin{array}{l}t_{2}^{*} \text { days } \\
\ldots .\end{array}$ & $\begin{array}{l}t_{1}^{*} \text { days } \\
\ldots \ldots\end{array}$ & $\begin{array}{l}Z^{*}(\$) \\
\ldots \ldots \ldots\end{array}$ \\
\hline 16 & 0.626 & 130.782 & 21.797 & 10 & 2 & 144.29 \\
\hline 20 & 1.216 & 65.364 & 10.894 & & & 665.62 \\
\hline 24 & 1.480 & 43.378 & 7.230 & & & 821.19 \\
\hline 28 & 1.665 & 32.495 & 5.416 & & & 889.72 \\
\hline 32 & 1.811 & 26.039 & 4.340 & & & 925.87 \\
\hline 36 & 1.933 & 21.787 & 3.631 & & & 947.31 \\
\hline 40 & 2.038 & 18.785 & 3.131 & & & 961.34 \\
\hline
\end{tabular}

From above table, we see that, if we set the shortage period to 2.4 days then the maximum profit will occur for maximum selling price $\$ 40.00$ (so obtained by treating the objective function as the functions of $\rho$ and $s_{1}$ only) which is very unrealistic.

The solutions in Table 4 are similar to the solution of Table 3.

Example 2: For Case-1, let $\eta=50$ units and $\tau=70$ units. If the set up cost $c_{1}=\$ 28.0$, holding cost per unit item $c_{2}=\$ 20.0$, shortage cost $c_{3}=\$ 100.0$, selling price per unit item $s_{1 \max }=\$ 40.0, s_{1 \mathrm{~min}}=\$ 12.0$, unit purchasing price $c_{1}=\$ 12.0$, publicity cost $k=\$ 15.0, m=3$, the cycle time $\mathrm{T}=0.4$ month and

$$
\rho_{0}=1.2, Z_{0}=129, s_{10}=23, p_{01}=.7, p_{02}=2.4 \text { and } p_{03}=716
$$

for GFO problem and, for IFO problem, we consider

$$
\begin{aligned}
& s_{1 U}^{r e j}=s_{1 U}^{a c c}=40, s_{1 L}^{a c c}=16, s_{1 L}^{r e j}=20, \rho_{L}^{a c c}=0.5, \rho_{L}^{r e j}=0.8 \\
& \rho_{U}^{r e j}=\rho_{U}^{a c c}=1.2, L^{a c c}=L^{r e j}=129, U^{a c c}=845.0, U^{r e j}=840.0 .
\end{aligned}
$$

Then, we have the solution Table 5.

Example 3: For Case-2, we consider the same data set (as per Case-1) along with

$$
\rho_{0}=3.6, Z_{0}=581, s_{10}=23, p_{01}=2.4, p_{02}=2.4 \text { and } p_{03}=264
$$


Table 5 Solution for $s_{1} \in\left[s_{1 \min }, s_{1 \max }\right]$ and $\rho \in\left[\rho_{\min }, \rho^{*}\right]$

\begin{tabular}{llllllllll}
\hline Type & $t_{2}^{*}$ & $t_{1}^{*}$ & $s^{*}$ & $q^{*}$ & $s_{1}^{*}$ & $\rho^{*}$ & $Z^{*}$ & $\alpha^{*}$ & $\beta^{*}$ \\
\hline GFO & 10 & 2 & 8.733 & 52.398 & 21.13 & 0.656 & 685.65 & 0.777 & $\ldots$ \\
IFO & 10 & 2 & 8.156 & 48.936 & 21.83 & 0.746 & 718.49 & 0.823 & .171 \\
\hline
\end{tabular}

for GFO problem and, for IFO problem, we assume

$$
\begin{aligned}
& s_{1 U}^{r e j}=s_{1 U}^{a c c}=40, s_{1 L}^{a c c}=16, s_{1 L}^{r e j}=20, \rho_{L}^{a c c}=1.2, \rho_{L}^{r e j}=1.6, \\
& \rho_{U}^{r e j}=\rho_{U}^{a c c}=3.6, L^{a c c}=L^{r e j}=581, U^{a c c}=845.0, U^{r e j}=841.0 .
\end{aligned}
$$

Then, we have the solution Table 6 .

Example 4 : For Case-3, we consider the same data set (as per Case-1) along with the assumptions $\rho_{0}=2.2, Z_{0}=2.0, s_{10}=23, p_{01}=1.0, p_{02}=2.4$ and $p_{03}=955$ for GFO problem and, for IFO problem, we assume

$$
\begin{aligned}
& s_{1 U}^{r e j}=s_{1 U}^{a c c}=40, s_{1 L}^{a c c}=16, s_{1 L}^{r e j}=20, \rho_{L}^{a c c}=1.2, \rho_{L}^{r e j}=1.6, \\
& \rho_{U}^{r e j}=\rho_{U}^{a c c}=3.6, L^{a c c}=L^{r e j}=2.0, U^{a c c}=957.0, U^{r e j}=950.0 .
\end{aligned}
$$

Then, we have the solution Table 7.

Example 5: For Case-4, we consider the same data set (as per Case-1) along with the assumptions $\rho_{0}=3.6, Z_{0}=2.0, s_{10}=23, p_{01}=1.4, p_{02}=2.4$ and $p_{03}=955$ for GFO problem and, for IFO problem, we assume

$$
\begin{aligned}
& s_{1 U}^{r e j}=s_{1 U}^{a c c}=40, s_{1 L}^{a c c}=16, s_{1 L}^{r e j}=20, \rho_{L}^{a c c}=2.2, \rho_{L}^{r e j}=2.6, \\
& \rho_{U}^{r e j}=\rho_{U}^{a c c}=3.6, L^{a c c}=L^{r e j}=2.0, U^{a c c}=957.0, U^{r e j}=950.0 .
\end{aligned}
$$

Then, we have the solution Table 8 .

\subsection{Interpretation on GFO and IFO Solutions (Tables 6, 7, 8)}

From Table 5, we see that, if $\rho$ assumes value 0.656 and unit selling price $s_{1}$ be $\$ 21.13$ then the average maximum profit is $\$ 685.65$ under GFO policy, but in IFO policy, the profit

Table 6 Solution for $s_{1} \in\left[s_{1 \min }, s_{1 \max }\right]$ and $\rho \in\left[\rho^{*}, \rho_{\max }\right]$

\begin{tabular}{llllllllll}
\hline Type & $t_{2}^{*}$ & $t_{1}^{*}$ & $s^{*}$ & $q^{*}$ & $s_{1}^{*}$ & $\rho^{*}$ & $Z^{*}$ & $\alpha^{*}$ & $\beta^{*}$ \\
\hline GFO & 10 & 2 & 8.272 & 49.634 & 22.91 & 1.891 & 768.97 & 0.712 & $\ldots$ \\
IFO & 10 & 2 & 7.918 & 47.505 & 23.42 & 1.942 & 780.90 & 0.753 & .247 \\
\hline
\end{tabular}

Table 7 Solution for $s_{1} \in\left[s_{1 \min }, s_{1 \max }\right]$ and $\rho \in\left[\rho^{*}, \rho^{\prime}\right]$

\begin{tabular}{llllllllll}
\hline Type & $t_{2}^{*}$ & $t_{1}^{*}$ & $s^{*}$ & $q^{*}$ & $s_{1}^{*}$ & $\rho^{*}$ & $Z^{*}$ & $\alpha^{*}$ & $\beta^{*}$ \\
\hline GFO & 10 & 2 & 9.267 & 55.600 & 21.47 & 1.427 & 739.34 & 0.772 & $\ldots$ \\
IFO & 10 & 2 & 8.503 & 51.018 & 22.31 & 1.476 & 771.36 & 0.804 & .196
\end{tabular}


Table 8 Solution for $s_{1} \in\left[s_{1 \min }, s_{1 \max }\right]$ and $\rho \in\left[\rho^{*}, \rho^{\prime}\right]$

\begin{tabular}{llllllllll}
\hline Type & $t_{2}^{*}$ & $t_{1}^{*}$ & $s^{*}$ & $q^{*}$ & $s_{1}^{*}$ & $\rho^{*}$ & $Z^{*}$ & $\alpha^{*}$ & $\beta^{*}$ \\
\hline GFO & 10 & 2 & 8.128 & 48.766 & 23.56 & 2.640 & 656.31 & 0.685 & $\ldots$ \\
IFO & 10 & 2 & 7.307 & 43.841 & 24.96 & 2.795 & 657.20 & 0.684 & .316 \\
\hline
\end{tabular}

Table 9 Sensitivity analysis for GFO problem

\begin{tabular}{|c|c|c|c|c|c|c|c|c|c|c|}
\hline \multicolumn{2}{|c|}{ Parameter $\%$ change } & \multirow{2}{*}{$\frac{t_{2}^{*}}{10}$} & \multirow{2}{*}{$\frac{t_{1}^{*}}{2}$} & \multirow{2}{*}{$\frac{s^{*}}{8.170}$} & \multirow{2}{*}{$\frac{q^{*}}{49.024}$} & \multirow{2}{*}{$\frac{s_{1}^{*}}{21.24}$} & \multirow{2}{*}{$\frac{\rho^{*}}{0.431}$} & \multirow{2}{*}{$\begin{array}{l}Z^{*} \\
653.11\end{array}$} & \multirow{2}{*}{$\frac{\alpha^{*}}{0.732}$} & \multirow{2}{*}{$\frac{\frac{Z^{*}-Z_{*}}{Z_{*}} \times 100 \%}{-4.70}$} \\
\hline$p_{01}$ & +50 & & & & & & & & & \\
\hline & +30 & 10 & 2 & 8.404 & 50.425 & 21.19 & 0.516 & 667.06 & 0.751 & -2.70 \\
\hline & -30 & 10 & 2 & 9.028 & 54.170 & 21.08 & 0.809 & 700.77 & 0.799 & +2.20 \\
\hline & -50 & 10 & 2 & 9.204 & 55.221 & 21.06 & 0.917 & 708.63 & 0.809 & +3.35 \\
\hline \multirow[t]{4}{*}{$p_{02}$} & +50 & 10 & 2 & 9.749 & 58.495 & 20.34 & 0.683 & 657.66 & 0.738 & -4.08 \\
\hline & +30 & 10 & 2 & 9.338 & 56.027 & 20.64 & 0.672 & 669.78 & 0.755 & -2.36 \\
\hline & -30 & 10 & 2 & 8.152 & 48.914 & 21.66 & 0.642 & 699.78 & 0.797 & +2.06 \\
\hline & -50 & 10 & 2 & 7.783 & 46.698 & 22.03 & 0.634 & 707.95 & 0.809 & +3.25 \\
\hline \multirow[t]{4}{*}{$p_{03}$} & +50 & 10 & 2 & 8.407 & 50.440 & 21.68 & 0.814 & 721.67 & 0.552 & +5.25 \\
\hline & +30 & 10 & 2 & 8.600 & 51.059 & 21.50 & 0.763 & 710.57 & 0.624 & +3.63 \\
\hline & -30 & - & - & - & \multicolumn{3}{|c|}{ no feasible solution } & - & - & - \\
\hline & -50 & - & - & - & \multicolumn{3}{|c|}{ no feasible solution } & - & - & - \\
\hline \multirow[t]{4}{*}{$s_{10}$} & +50 & 10 & 2 & 3.019 & 18.766 & 32.56 & 0.636 & 706.36 & 0.806 & +3.02 \\
\hline & +30 & 10 & 2 & 4.311 & 25.866 & 27.86 & 0.606 & 736.86 & 0.849 & +7.46 \\
\hline & -30 & $\cdots \cdot$ & ...no & feasible & solution. &.. & & & & - \\
\hline & -50 & $\cdots$ & ...no & feasible & solution. & & & & & - \\
\hline \multirow[t]{4}{*}{$\rho_{0}$} & +50 & 10 & 2 & 9.581 & 57.688 & 21.02 & 1.222 & 719.83 & 0.825 & +4.98 \\
\hline & +30 & 10 & 2 & 9.308 & 55.848 & 21.04 & 0.989 & 712.72 & 0.815 & +3.94 \\
\hline & -30 & 10 & 2 & 7.897 & 47.384 & 21.30 & 0.344 & 636.20 & 0.708 & -7.20 \\
\hline & -50 & 10 & 2 & 7.144 & 42.865 & 21.46 & 0.152 & 587.02 & 0.639 & -14.38 \\
\hline \multirow[t]{4}{*}{$Z_{0}$} & +50 & 10 & 2 & 8.625 & 51.750 & 21.31 & 0.707 & 697.86 & 0.704 & +1.78 \\
\hline & +30 & 10 & 2 & 8.668 & 52.008 & 21.24 & 0.686 & 693.03 & 0.734 & +1.07 \\
\hline & -30 & 10 & 2 & 8.798 & 52.790 & 21.03 & 0.625 & 678.11 & 0.820 & -1.09 \\
\hline & -50 & 10 & 2 & 8.842 & 53.053 & 20.96 & 0.605 & 673.00 & 0.849 & -1.80 \\
\hline
\end{tabular}

increases to $\$ 718.49$ with a little variation in $\rho$ and $s_{1}$ alone. From Table 6 we see, though the profit function is decreasing in $\rho$ but at $\rho=1.942$ we have received a better result in IFO policy for $s_{1}=\$ 23.42$. However the Table 7 shows, in GFO policy, the average profit is quite high (\$20.85) with $\rho=1.427$ which is approximately double of the value of IFO policy with respect to the Table 5. Also, we see in IFO policy if $\rho$ assumes values near 1.5 and $s_{1}=\$ 22.31$ then the average profit be slightly less $(\$ 9.55)$ for the benefit in $\rho=0.47$ alone with respect to the Table 6 for both the cases of GFO and IFO policies. Again from Table 8 , we have noticed that though both unit selling price $s_{1}$ and promotional index $\rho$ are very high, but the average profit is very much depressive and it is below the results of the crisp one. 


\section{Maximum Profit Z}

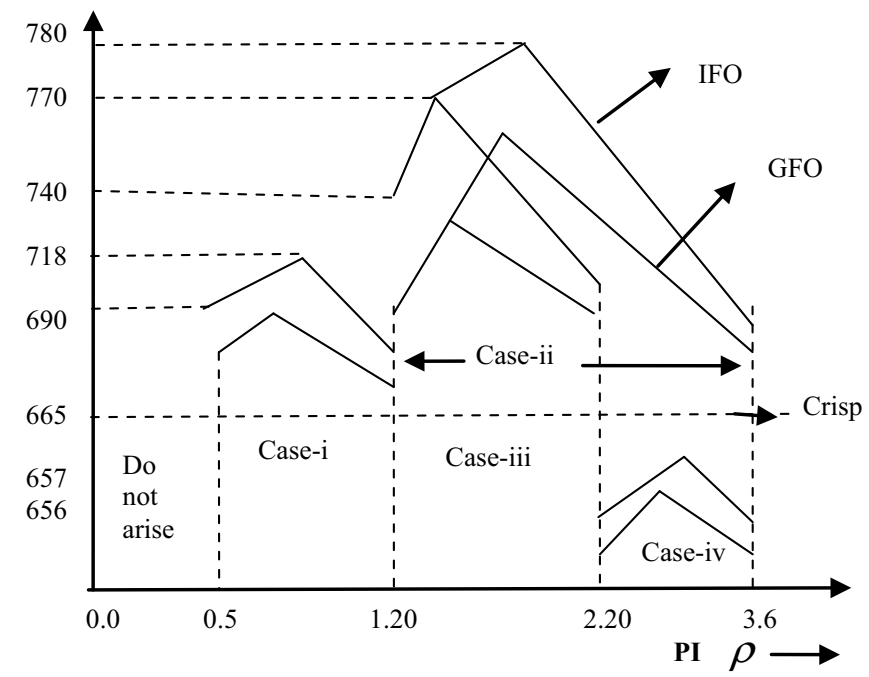

Fig. 4 GFO and IFO solutions of the model

\subsection{Sensitivity Analysis of GFO Model (Referring to Table 5)}

The sensitivity of the parameters $\left\{p_{01}, p_{02} \quad p_{03}, s_{10}, \rho_{0}\right.$ and $\left.Z_{0}\right\}$ from $-50 \%$ to $+50 \%$ changes for the GFO model which is shown in the following table.

\subsection{Sensitivity Analysis of IFO Model (Referring to Table 5)}

The sensitivity analysis of the parameters $\left\{s_{1 L}^{r e j} \& s_{1 U}^{a c c}, s_{1 L}^{r e j}, s_{1 L}^{a c c}, \rho_{U}^{r e j} \& \rho_{U}^{a c c}, \rho_{L}^{a c c}, \rho_{L}^{r e j}\right.$, $L^{a c c} \& L^{r e j}, U^{a c c}$ and $\left.U^{r e j}\right\}$ from $-50 \%$ to $+50 \%$ changes for the IFO model which is shown in the following table.

\subsection{Comments on Sensitivity Analysis for GFO and IFO Problems}

From Table 9, we see that all the parametric values are poorly sensitive or have the insensitivity for the GFO model. Only one case arises where $\rho_{0}$ changes to $-50 \%$ giving average sensitivity to $-14.38 \%$. On the other hand, the Table 10 shows that all the parameters have insensitivity that means no effects can be carried out whenever any kind of tolerance is made in the IFO model. Note that this sensitivity analysis has been done to study the trend solution of the models so that we feel no need the same for the Tables 6, 7, 8 also.

\subsection{Comments over the Graph of the Model}

As per Table 4, $s_{1}$ assumes values in $[16,40]$ and Fig. 4 shows optimum profit function where, in all cases, IFO policy gives better result than GFO policy. The upper line bar shows the optimal solutions for the IFO policy and the lower line bar in each interval shows the solutions for GFO policy. If PE lies in $[1.2,3.6]$, then the average maximum profit will occur in Case-2. Splitting the interval into $[1.2,2.2]$ and $[2.2,3.6]$, we see the average profit is unsatisfactory in both the cases for GFO and IFO policies in $[2.2,3.6]$. This result lies below 
Table 10 Sensitivity analysis for IFO problem

\begin{tabular}{|c|c|c|c|c|c|c|c|c|c|c|c|}
\hline $\begin{array}{l}\text { Parameter } \\
\% \text { change }\end{array}$ & & $t_{2}^{*}$ & $t_{1}^{*}$ & $s^{*}$ & $q^{*}$ & $s_{1}^{*}$ & $\rho^{*}$ & $Z^{*}$ & $\alpha^{*}$ & $\beta^{*}$ & $\begin{array}{l}\frac{Z^{*}-Z_{*}}{Z_{*}} \\
\times 100 \%\end{array}$ \\
\hline \multirow[t]{4}{*}{$s_{1 L}^{r e j} \& s_{1 U}^{a c c}$} & +50 & 10 & 2 & 6.756 & 40.538 & 23.48 & 0.722 & 749.57 & 0.866 & 0.127 & +4.32 \\
\hline & +30 & 10 & 2 & 7.222 & 43.333 & 22.87 & 0.729 & 740.46 & 0.853 & 0.140 & +3.05 \\
\hline & -30 & 10 & 2 & 9.805 & 58.831 & 20.42 & 0.783 & 670.44 & 0.756 & 0.238 & -6.70 \\
\hline & -50 & & o fe & le soluti & on....... & & & & & & - \\
\hline \multirow[t]{4}{*}{$s_{1 L}^{r e j}$} & +50 & 10 & 2 & 5.834 & 35.004 & 24.91 & 0.712 & 763.05 & 0.889 & 0.108 & +6.20 \\
\hline & +30 & 10 & 2 & 6.749 & 40.494 & 23.49 & 0.722 & 749.71 & 0.866 & 0.127 & +4.34 \\
\hline & -30 & - & - & - & \multicolumn{6}{|c|}{ no feasible solution } & - \\
\hline & -50 & - & - & - & \multicolumn{6}{|c|}{ no feasible solution } & - \\
\hline \multirow[t]{4}{*}{$s_{1 L}^{a c c}$} & +50 & 10 & 2 & 6.297 & 37.785 & 24.15 & 0.716 & 757.11 & 0.877 & 0.117 & +5.37 \\
\hline & +30 & 10 & 2 & 7.039 & 42.235 & 23.10 & 0.726 & 744.20 & 0.859 & 0.135 & +3.57 \\
\hline & -30 & 10 & 2 & 9.217 & 55.301 & 20.87 & 0.769 & 688.72 & 0.781 & 0.213 & -4.14 \\
\hline & -50 & 10 & 2 & 9.874 & 59.245 & 20.37 & 0.785 & 668.22 & 0.753 & 0.242 & -7.00 \\
\hline \multirow[t]{4}{*}{$\rho_{U}^{r e j} \& \rho_{U}^{a c c}$} & +50 & 10 & 2 & 8.461 & 50.768 & 21.66 & 0.841 & 723.99 & 0.831 & 0.163 & +0.80 \\
\hline & +30 & 10 & 2 & 8.348 & 50.091 & 21.72 & 0.804 & 722.03 & 0.828 & 0.166 & +0.50 \\
\hline & -30 & 10 & 2 & 7.927 & 47.563 & 21.97 & 0.685 & 714.01 & 0.817 & 0.177 & -0.60 \\
\hline & -50 & $\ldots$ & o fe & le soluti & on....... & $\cdots$ & 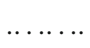 & $\ldots \ldots \ldots$ & & & \\
\hline \multirow[t]{4}{*}{$\rho_{L}^{a c c}$} & +50 & 10 & 2 & 8.473 & 50.839 & 21.65 & 0.846 & 724.20 & 0.831 & 0.163 & +0.80 \\
\hline & +30 & 10 & 2 & 8.353 & 50.115 & 21.72 & 0.805 & 722.10 & 0.828 & 0.166 & +0.50 \\
\hline & -30 & 10 & 2 & 7.940 & 47.642 & 21.96 & 0.688 & 714.28 & 0.817 & 0.177 & -0.60 \\
\hline & -50 & 10 & 2 & 7.786 & 46.714 & 22.06 & 0.650 & 711.10 & 0.813 & 0.181 & -1.01 \\
\hline \multirow[t]{4}{*}{$\rho_{L}^{r e j}$} & +50 & 10 & 2 & 8.642 & 51.851 & 21.57 & 0.908 & 726.97 & 0.835 & 0.159 & +1.18 \\
\hline & +30 & 10 & 2 & 8.463 & 50.700 & 21.66 & 0.842 & 724.03 & 0.831 & 0.163 & +0.80 \\
\hline & -30 & 10 & 2 & 7.799 & 46.793 & 22.05 & 0.653 & 711.38 & 0.813 & 0.181 & -1.01 \\
\hline & -50 & $\ldots$ & o fe & le soluti & on.... & . & & $\ldots \ldots \ldots$ & & & - \\
\hline \multirow[t]{4}{*}{$L^{a c c} \& L^{r e j}$} & +50 & 10 & 2 & 7.985 & 47.915 & 22.02 & 0.751 & 724.20 & 0.815 & 0.179 & +0.80 \\
\hline & +30 & 10 & 2 & 8.055 & 48.334 & 21.94 & 0.749 & 721.87 & 0.818 & 0.176 & +0.50 \\
\hline & -30 & 10 & 2 & 8.251 & 49.510 & 21.73 & 0.743 & 715.25 & 0.827 & 0.166 & -0.60 \\
\hline & -50 & 10 & 2 & 8.313 & 49.879 & 21.66 & 0.742 & 713.15 & 0.831 & 0.164 & -0.70 \\
\hline \multirow[t]{4}{*}{$U^{a c c}$} & +50 & 10 & 2 & 6.471 & 38.825 & 24.12 & 0.820 & 774.46 & 0.567 & 0.092 & +7.78 \\
\hline & +30 & 10 & 2 & 6.962 & 41.774 & 23.34 & 0.795 & 758.94 & 0.650 & 0.114 & +5.62 \\
\hline & -30 & - & - & - & \multicolumn{6}{|c|}{ no feasible solution ...... } & - \\
\hline & -50 & - & - & - & \multicolumn{6}{|c|}{ no feasible solution..$\ldots \ldots \ldots \ldots$} & - \\
\hline \multirow[t]{4}{*}{$U^{r e j}$} & +50 & - & - & - & \multicolumn{6}{|c|}{ no feasible solution . ....... } & - \\
\hline & +30 & .. & ... & & \multicolumn{6}{|c|}{ no feasible solution . ............. } & - \\
\hline & -30 & 10 & 2 & 9.292 & 55.755 & 20.76 & 0.730 & 680.39 & 0.770 & 0.000 & -5.30 \\
\hline & -50 & 10 & 2 & 9.292 & 55.755 & 20.76 & 0.730 & 680.39 & 0.770 & 0.000 & -5.30 \\
\hline
\end{tabular}


Table 11 Sensitivity analysis

\begin{tabular}{|c|c|c|c|c|c|c|c|c|}
\hline \multicolumn{2}{|c|}{ Parameter \% change } & \multirow{2}{*}{$\frac{\rho^{*}}{1.216}$} & \multirow{2}{*}{$\frac{q^{*}}{65.364}$} & \multirow{2}{*}{$\frac{s^{*}}{10.874}$} & \multirow{2}{*}{$\frac{t_{1}^{*}}{10.0}$} & \multirow{2}{*}{$\frac{t_{2}^{*}}{2.0}$} & \multirow{2}{*}{$\frac{Z_{*}}{630.62}$} & \multirow{2}{*}{$\frac{\frac{Z_{*}-Z^{*}}{Z^{*}} 100 \%}{-5.26}$} \\
\hline$c_{1}$ & +50 & & & & & & & \\
\hline & +30 & 1.216 & 65.364 & 10.874 & 10.0 & 2.0 & 644.62 & -3.15 \\
\hline & -30 & 1.216 & 65.364 & 10.874 & 10.0 & 2.0 & 686.62 & 3.15 \\
\hline & -50 & 1.216 & 65.364 & 10.874 & 10.0 & 2.0 & 700.62 & 5.26 \\
\hline \multirow[t]{4}{*}{$c_{2}$} & +50 & 1.095 & 64.636 & 14.916 & 9.0 & 3.0 & 457.28 & -31.30 \\
\hline & +30 & 1.145 & 64.944 & 13.401 & 9.5 & 2.5 & 536.33 & -19.42 \\
\hline & -30 & 1.285 & 65.747 & 8.074 & 10.5 & 1.5 & 809.39 & 21.60 \\
\hline & -50 & 1.331 & 65.986 & 6.000 & 11.0 & 1.0 & 914.45 & 37.38 \\
\hline \multirow[t]{4}{*}{$c_{3}$} & +50 & 1.199 & 65.268 & 7.679 & 10.6 & 1.4 & 633.60 & -4.81 \\
\hline & +30 & 1.205 & 65.299 & 8.707 & 10.4 & 1.6 & 643.84 & -3.27 \\
\hline & -30 & 1.234 & 65.468 & 14.548 & 9.0 & 3.0 & 701.96 & 5.46 \\
\hline & -50 & 1.255 & 65.581 & 18.737 & 8.6 & 3.4 & 743.50 & 11.70 \\
\hline \multirow[t]{4}{*}{$\eta$} & +50 & 1.216 & 90.364 & 15.394 & 10.0 & 2.0 & 957.28 & 43.82 \\
\hline & +30 & 1.216 & 80.364 & 13.394 & 10.0 & 2.0 & 840.62 & 26.29 \\
\hline & -30 & 1.216 & 50.364 & 8.394 & 10.0 & 2.0 & 490.62 & -26.29 \\
\hline & -50 & 1.216 & 40.364 & 6.727 & 10.0 & 2.0 & 373.95 & -43.82 \\
\hline \multirow[t]{4}{*}{$\tau$} & +50 & 1.384 & 74.383 & 12.397 & 10.0 & 2.0 & 758.03 & 13.88 \\
\hline & +30 & 1.322 & 70.728 & 11.788 & 10.0 & 2.0 & 720.46 & 8.24 \\
\hline & -30 & 1.082 & 60.188 & 10.031 & 10.0 & 2.0 & 613.17 & -7.88 \\
\hline & -50 & 0.968 & 56.886 & 9.481 & 10.0 & 2.0 & 580.07 & -12.85 \\
\hline \multirow[t]{4}{*}{$s_{1}$} & +50 & 1.742 & 28.899 & 4.817 & 10.0 & 2.0 & 910.36 & 36.77 \\
\hline & +30 & 1.580 & 37.144 & 6.191 & 10.0 & 2.0 & 861.43 & 29.42 \\
\hline & -30 & $\ldots \ldots n$ & feasible & lution.... & & & $\ldots \ldots$ & ....... \\
\hline & -50 & $\ldots \ldots$ & feasible & lution... & & $\ldots \ldots$ & $\ldots$ & $\ldots$ \\
\hline \multirow[t]{4}{*}{$p_{1}$} & +50 & $\ldots \ldots \ldots$ & feasible & lution & & $\ldots$ & $\ldots \ldots \ldots$ & $\ldots \ldots$ \\
\hline & +30 & 0.740 & 61.910 & 10.318 & 10.0 & 2.0 & 89.01 & -86.63 \\
\hline & -30 & 1.459 & 66.611 & 11.102 & 10.0 & 2.0 & 1260.09 & 89.31 \\
\hline & -50 & 1.579 & 67.144 & 11.191 & 10.0 & 2.0 & 1661.43 & 149.61 \\
\hline \multirow[t]{4}{*}{$k$} & +50 & 1.065 & 64.442 & 10.740 & 10.0 & 2.0 & 654.69 & -1.64 \\
\hline & +30 & 1.116 & 64.771 & 10.795 & 10.0 & 2.0 & 658.52 & -1.07 \\
\hline & -30 & 1.363 & 66.150 & 11.025 & 10.0 & 2.0 & 675.17 & 1.44 \\
\hline & -50 & 1.515 & 66.867 & 11.145 & 10.0 & 2.0 & 684.03 & 2.77 \\
\hline
\end{tabular}

the crisp optimal level. Finally we may say that to have a considerable average profit, we always enrich our $\rho$ values into the interval $[1.2,2.2]$ whatever the unit selling price within be $[16,40]$.

\section{Sensitivity Analysis of the Crisp Model}

The sensitivity for the crisp model of the parameters $\left\{c_{1}, c_{2}, c_{3}, \eta, \tau, s_{1}, p_{1}\right.$ and $\left.k\right\}$ changes from $(-50 \%$ to $+50 \%)$ is shown in the following table. 
From the Table 11, it is observed that the parameters $\left(\eta, s_{1}, \tau, c_{2}\right)$ are high sensitive whenever a change is made from $(-50 \%$ to $+50 \%)$ each separately. At $+50 \%$ change of the demand parameter $\eta$ the profit is increased to $43.82 \%$ and it decreases to $43.82 \%$, whenever a change is made to $-50 \%$. For shortage $\operatorname{cost} c_{2}$, at $+50 \%$ change, the profit decreases to $31.30 \%$ and, at $-50 \%$ change, it increases to $37.38 \%$. The cost price parameter $p_{1}$ and selling price parameter $s_{1}$ is extraordinarily high sensitive for their changes from $-50 \%$ to $+50 \%$. At $-50 \%$ change of $p_{1}$, the relative increment of the total average profit reaches to $149.61 \%$ and has no feasible solution for $+50 \%$ change. But at $+30 \%$ change for the cost price $p_{1}$, the average profit decreases to $-86.63 \%$. The other parameters $\left(c_{1}, \tau \quad c_{3}\right.$ and $\left.k\right\}$ are less sensitive with respect to the crisp optimal solution.

Throughout the whole table, the average total profit will be maximum when the cost price decreases to $-50 \%$ and, in that case, the decision variables are the order quantity $q^{*}=67.144$, the shortage quantity $\mathrm{s}^{*}=11.191$, the $\mathrm{PE} \rho^{*}=1.79$ and the maximum average profit $Z_{*}=\$ 1661.43$.

\section{Conclusion}

In this paper, we have discussed the nature of the average profit function for the classical backorder model under fuzzy promotional effort and fuzzy unit selling price. The demand rate is affected by unit selling price and the PE variable. From the crisp result or more specific from Table 4, the optimality reaches at maximum unit selling price which is the unfavorable situation in practice. In our study of GFO and IFO policy, we have incorporated the most common $(\beta) \quad \alpha$-cut of the fuzzy (non) membership function as well. To do this, we have considered the whole span of each interval to measure the tolerance values. However, with proper choice of upper and lower bounds, the fuzzy sets have been constructed in a nice way. Also, to get a comparative study within the models (Crisp, GFO and IFO), we have gone through some sensitivity analysis to each of the models. From the Tables 9, 10, 11, we observed that IFO policy is better to make a decision for a managerial part. Although, few deviations (poorly sensitivity) have been observed in GFO and IFO tables but a trend study shows such errors due to the human unconsciousness. From the whole observations, we have analyzed that neither more unit selling price nor less promotional cost would promote a maximum profit for the models. Therefore, a serious attention is required for all time to cope with the model itself.

The proposed model can be extended further incorporating time value of money and inflation of the cost parameters. Partial backlogging with variable shortage cost and delayin-payment policy may be considered in the present model for future extension.

\section{References}

1. Cárdenas-Barrón, L.E.: Optimizing inventory decisions in a multi stage multi-customer supply chain: a note. Trans. Res. Part E 43, 647-654 (2007)

2. Cárdenas-Barrón, L.E., Taleizadeh, A.A., Treviño-Garza, G.: An improved solution to replenishment lot size problem with discontinuous issuing policy and rework, and the multi-delivery policy into economic production lot size problem with partial rework. Expert Syst. Appl. 39, 3888-3895 (2012)

3. Cárdenas-Barrón, L.E., Treviño-Garza, G., Wee, H.M.: A simple and better algorithm to solve the vendor managed inventory control system of multi-product multi-constraint economic order quantity model. Expert Syst. Appl. 39, 3888-3895 (2012)

4. Cárdenas-Barrón, L.E., Treviño-Garza, G.: An optimal solution to a three echelon supply chain network with multi-product and multi-period. Appl. Math. Model. 38, 1911-1918 (2014) 
5. Cárdenas-Barrón, L.E., Porter, J.D.: Supply chain models for an assembly system with preprocessing of raw materials: a simple and better algorithm. Appl. Math. Model. 37, 7883-7887 (2013)

6. Sarkar, B.: An EOQ model with delay in payments and stock dependent demand in the presence of imperfect production. Appl. Math. Comput. 218, 8295-8308 (2012)

7. Sarkar, B.: An inventory model with reliability in an imperfect production process. Appl. Math. Comput. 218, 4881-4891 (2012)

8. Sarkar, B.: An EOQ model with delay-in-payments and time-varying deterioration rate. Math. Comput. Model. 55, 367-377 (2012)

9. Sarkar, B., Saren, S., Wee, H.M.: An inventory model with variable demand, component cost and selling price for deteriorating items. Econ. Model. 30, 306-310 (2013)

10. Sett, B.K., Sarkar, B., Goswami, A.: A two-warehouse inventory model with increasing demand and time varying deterioration. Scientia Iranica, Trans. E 19, 306-310 (2012)

11. Sana, S.S., Chaudhuri, K.S.: An inventory model for stock with advertising sensitive demand. IMA J. Manag. Math. 19, 51-62 (2008)

12. Sana, S.S.: Demand influenced by enterprises' initiatives - a multi-item EOQ model of deteriorating and ameliorating items. Math. Comput. Model. 52, 284-302 (2010)

13. Sana, S.S.: An EOQ model of homogeneous products while demand is salesmen's initiatives and stock sensitive. Comput. Math. Appl. 62, 577-587 (2011)

14. Sana, S.S.: An EOQ model for salesmen's initiatives, stock and price sensitive demand of similar products-a dynamical system. Appl. Math. Comput. 218, 3277-3288 (2011)

15. Sana, S.S.: The EOQ model—a dynamical system. Appl. Math. Comput. 218, 8736-8749 (2012)

16. Cárdenas-Barrón, L.E., Sana, S.S.: A production-inventory model for a two-echelon supply chain when demand is dependent on sales teamsx initiatives. Int. J. Prod. Econ. 155, 249-258 (2014)

17. Sana, S. S.: Optimal production lot size and reorder point of a two-stage supply chain while random demand is sensitive with sales teams' initiatives. International Journal of Systems Science. 1-16(2014). doi:10.1080/00207721.2014.886748

18. Sarkar, B., Sana, S., Chauhduri, K.S.: Optimal reliability, production lotsize and safety stock: an economic manufacturing quantity model. Int. J. Manag. Sci. Eng. Manag. 5, 192-202 (2010)

19. Sarkar, B., Cárdenas-Barrón, L.E., Sarkar, M., Singgih, M.L.: An economic production quantity model with random defective rate, rework process and backorders for a single stage production system. J. Manuf. Syst. 33, 423-435 (2014)

20. Sarkar, B., Sarkar, S.: An improved inventory model with partial backlogging, time varying deterioration and stock-dependent demand. Econ. Model. 30, 924-932 (2013)

21. Pal, B., Sana, S. S., Chaudhuri, K. S.: Optimal pricing and ordering policy for two echelon varying production inventory system. J. Ind. Eng. Article ID 429836, 11 pages (2014). doi:10.1155/2014/605178

22. De, S. K., Sana, S. S., Goswami, A.: An EOQ model for phase inventory with induced demand and periodic cycle time. J. Ind. Eng. Article ID 605178, 14 pages (2014). doi:10.1155/2014/429836

23. Sana, S.: An EOQ model for stochastic demand for limited capacity of own warehouse. Ann. Oper. Res. 1-17 (2013). doi:10.1007/s10479-013-1510-5

24. Sana, S. S., Goyal, S. K.: (Q, r, L) model for stochastic demand with lead-time dependent partial backlogging. Ann. Oper. Res. 1-10 (2014). doi:10.1007/s10479-014-1731-2

25. Zadeh, L.A.: Fuzzy sets. Inf. Control. 8, 338-356 (1965)

26. Bellman, R.E., Zadeh, L.A.: Decision making in a fuzzy environment. Manag. Sci. 17, B141-B164 (1970)

27. Kaufmann, A., Gupta, M.: Fuzzy mathematical models in engineering and management science. Elsevier Science Inc., New York (1988)

28. Vojosevic, M., Petrovic, D., Petrovic, R.: EOQ formula when inventory cost is fuzzy. Int. J. Prod. Econ. 45, 499-504 (1996)

29. Wu, K., Yao, J.S.: Fuzzy inventory with backorder for fuzzy order quantity and fuzzy shortage quantity. Eur. J. Oper. Res. 150, 320-352 (2003)

30. Lee, H.M., Yao, J.S.: Economic order quantity in fuzzy sense for inventory without back order model. Fuzzy Sets Syst. 105, 13-31 (1999)

31. Yao, J.S., Chang, S.C., Su, J.S.: Fuzzy inventory without backorder for fuzzy order quantity and fuzzy total demand quantity. Comput. Oper. Res. 27, 935-962 (2000)

32. Kao, C., Hsu, W.K.: Lot Size reorder point inventory model with fuzzy demands. Comput. Math. Appl. 43, 1291-1302 (2002)

33. De, S.K., Kundu, P.K., Goswami, A.: An economic production quantity inventory model involving fuzzy demand rate and fuzzy deterioration rate. J. Appl. Math. Comput. 12, 251-260 (2003)

34. De, S.K., Kundu, P.K., Goswami, A.: Economic ordering policy of deteriorated items with shortage and fuzzy cost co-efficients for vendor and buyer. Int. J. Fuzzy Syst. Rough Syst. 1, 69-76 (2008) 
35. Kumar, R.S., De, S.K., Goswami, A.: Fuzzy EOQ models with ramp type demand rate, partial backlogging and time dependent deterioration rate. Int. J. Math. Oper. Res. 4, 473-502 (2012)

36. Kabiran, A., Olafsson, S.: Continuous optimization via simulation using golden region search. Eur. J. Oper. Res. 208, 19-27 (2011)

37. Saleh, M., Oliva, R., Kampmann, C.E., Davidson, P.I.: A comprehensive analytical approach for policy analysis of system dynamics models. Eur. J. Oper. Res. 203, 673-683 (2010)

38. Atanassov, K.: Intuitionistic fuzzy sets and system. Fuzzy sets Syst. 20, 87-96 (1986)

39. Angelov, P.P.: Optimization in an intuitionistic fuzzy environment. Fuzzy sets Syst. 86, 299-306 (1997)

40. Atanassov, K., Gargov, G.: Interval valued intuitionistic fuzzy sets. Fuzzy sets Syst. 31, 343-349 (1989)

41. Banerjee, S., Roy, T.K.: Solution of a probabilistic fixed order interval system: a general fuzzy programming technique and intuitionistic fuzzy optimization technique. Glob. J. Financ. Manag. 2, 257-294 (2010)

42. De, S.K., Sana, S.S.: Fuzzy order quantity inventory model with fuzzy shortage quantity and fuzzy promotional index. Econ. Model. 31, 351-358 (2013)

43. De, S. K., Sana, S. S.: Backlogging EOQ model for promotional effort and selling price sensitive demandan intuitionistic fuzzy approach. Ann. Oper. Res. 1-20 (2013). doi:10.1007/s10479-013-1476-3

44. De, S.K., Goswami, A., Sana, S.S.: An interpolating by pass to pareto optimality in intuitionistic fuzzy technique for a EOQ model with time sensitive backlogging. Appl. Math. Comput. 230, 664-674 (2014)

45. De, S.K., Sana, S.S.: A multi-periods production-inventory model with capacity constraints for multimanufacturers-a global optimality in intuitionistic fuzzy environment. Appl. Math. Comput. 242, 825-841 (2014)

46. Das, P., De, S., Sana, S.S.: An EOQ model for time dependent backlogging over idle time: a step order fuzzy approach. Int. J. Appl. Comput. Math. (2014). doi:10.1007/s40819-014-0001-y

47. Zimmermann, H.J.: Fuzzy linear programming with several objective functions. Fuzzy sets Syst. 1, 46-55 (1978) 